\title{
Epilepsie liée aux accidents de la voie publique chez les Guinéens en 2012
}

\author{
Epilepsy linked to road accidents among Guineans in 2012
}

\author{
Diarra $\mathrm{AS}^{1}$, Dansoko DD¹, Bocoum FA¹, Guilavogui $\mathrm{V}^{2}$, Beavogui $\mathrm{KL}^{2}$.
}

\begin{abstract}
1 Département d'Etude et de Recherche en Santé Publique du Mali 2 Hôpital National Donka, service de neurochirurgie, BP 234 Conakry Guinée

Correspondant : Dr Diaba Denise DANSOKO s/c DERSP, Tel : 6699 34 82, diabadenise@gmail.com
\end{abstract}

\section{Résumé}

Introduction : L'épilepsie post-traumatique est la survenue d'une ou plusieurs crises épileptiques au-delà de la première semaine suivant un traumatisme crânien. Les séquelles invalidantes qu'engendrent cette pathologie, les difficultés de prise en charge, l'augmentation des accidents de la voie publique ont motivé notre étude. Son but était d'étudier la place des accidents de la voie publique dans les épilepsies pour contribuer à l'amélioration de sa prise en charge et la sécurité routière en Guinée en 2012. Méthode : C'était une étude transversale de Janvier 2010 à Décembre 2012 qui a porté sur 21 dossiers de malades hospitalisés pour traumatisme cranio encéphalique ayant développé une EPT. Les données du questionnaire ont été analysées sur Epi Info version 3.5.1, les iconographies interprétées par un spécialiste. Résultat : La prévalence de l'affection était de 1,6\% parmi les hospitalisés (21/1304) avec une prédominance masculine $66,7 \%$ et un sex ratio de 2. Les accidents de la voie publique étaient les circonstances de survenue les plus importantes avec $81 \%$. A l'admission $90,5 \%$ des patients avaient un trouble de la vigilance, $52,4 \%$ un Traumatisme crânien sévère, $57,1 \%$ une crise tonico-clonique, $61,9 \%$ un point d'impact frontal, $85,7 \%$ une anomalie focalisée à l'électroencéphalogramme et $95,2 \%$ ont eu une amélioration de l'état clinique avant la sortie. Conclusion L'élément préventif sûr de l'Epilepsie Post Traumatique reste la lutte contre les traumatismes crâniens en insistant sur l'éducation routière, en impliquant les médias dans la stratégie de sensibilisation et en améliorant les conditions de sécurité routière.

Mots clés: Epilepsie post traumatique, traumatisme crânien, Guinée

\footnotetext{
Abstract:

Introduction: Post-traumatic epilepsy is the occurrence of one or more epileptic seizures beyond the first week following a head trauma. The disabling sequelae caused by this pathology, the difficulties of treatment, the increase in accidents on the public highway motivated our study. Its aim was to study the place of accidents on the public highway in epilepsy to contribute to the improvement of its management and road safety in Guinea in 2012. Method: It was a cross-sectional study from January 2010 to December 2012 which focused on 21 files of patients hospitalized for cranio encephalic trauma having developed EFA. The questionnaire data were analyzed on Epi Info
}

version 3.5.1, the iconographies interpreted by a specialist. Result: The prevalence of the disease was $1.6 \%$ among hospitalized patients $(21 / 1304)$ with a male prevalence of $66.7 \%$ and a sex ratio of 2 . Accidents on the public highway were the most important circumstances with $81 \%$. On admission $90.5 \%$ of patients had an alertness disorder, $52.4 \%$ had a severe head trauma, $57.1 \%$ had a tonic-clonic attack, $61.9 \%$ had a frontal impact point, $85.7 \%$ a focused anomaly on the electroencephalogram and $95.2 \%$ had an improvement in the clinical state before discharge. Conclusion The sure preventive element of Post Traumatic Epilepsy remains the fight against head trauma by insisting on road education, by involving the media in the awareness raising strategy and by improving road safety conditions.

Keywords: Post traumatic epilepsy, head trauma, Guinea

\section{INTRODUCTION :}

L'épilepsie post-traumatique (EPT) est la survenue d'une ou plusieurs crises d'épilepsie au-delà de la première semaine suivant un traumatisme crânien (TC).

Pour affirmer EPT, il faut que le TC précède les crises mais aussi qu'il en soit la cause.

Les crises précoces de moins de $24 \mathrm{H}$ ou retardées de plus d'une semaine sont des crises symptomatiques aigues correspondant à la réaction du cerveau aux effets physiques du TC. Les crises secondaires ou tardives survenant après la première semaine répondent à la définition de la maladie épileptique post traumatique (1).

Dans le monde, les études épidémiologiques retrouvent que l'EPT représente $20 \%$ des épilepsies symptomatiques dans la population générale et $5 \%$ en consultation spécialisée d'épilepsie (2).

L'incidence de l'EPT est difficile à apprécier à cause de la très grande diversité de la notion TC. Dans les séries (populations) militaires, on observe des proportions concordantes de 30 à $40 \%$ en cas de plaies craniocérébrales (3). Dans les séries (populations) civiles, en raison notamment de la rareté des plaies craniocérébrales, l'incidence est nettement plus faible, de l'ordre de 3 à $7 \%$, voire moins si l'on inclut les TC bénins dans les statistiques (4).

En Afrique, le traumatisme crânien constitue la cause de l'épilepsie chez $14 \%$ des enfants de moins de 14 ans, 30\% des jeunes âgés de 15 à 34 ans, et $8 \%$ des adultes de plus de 65 ans (5).

Les facteurs de risque responsable de l'épilepsie post traumatique sont les plaies crânio-cérébrales, les embarrures associées à des hématomes extra ou intracérébraux, les lésions focales intracérébrales et l'amnésie post traumatique supérieure à $24 \mathrm{~h}$. 
En fonction de la gravité du traumatisme crânien, l'incidence cumulative de l'épilepsie est de 2,1\% après un TC léger ; $4,2 \%$ après un TC modéré et de $16,7 \%$ après un TC sévère (5).

En Guinée, les TC constituent un problème majeur de santé publique du fait de leur fréquence, leur gravité, et surtout de leurs séquelles lourdes. Les accidents de la voie publique (AVP) restent la première cause des TC dans toutes les catégories de gravité (6). Les accidents de la voie publique entrainent très souvent des épilepsies soit immédiates ou tardives et qui passent souvent inaperçues dans les services de santé.

Les séquelles invalidantes qu'engendrent cette pathologie, sa stigmatisation, les difficultés de prise en charge, l'augmentation des accidents de la voie publique et le besoin de ralentir ce fléau ont motivé notre étude. Elle avait pour but d'étudier la place des accidents de la voie publique dans la survenue des épilepsies afin de contribuer à l'amélioration de la sécurité routière et de la prise en charge de l'EPT à l'hôpital de Donka en Guinée. C'est dans ce cadre que la présente étude «Epilepsie liée aux accidents de la voie publique chez les Guinéens en 2012 » a été initiée.

\section{MATERIEL METHODES :}

Notre étude transversale s'est déroulée dans le service de Neurochirurgie de l'hôpital national Donka de janvier 2010 à 31 décembre 2012 et a porté sur 21 dossiers des malades hospitalisés pour TCE qui ont développé une EPT.

Matériels utilisés : le registre d'hospitalisation, les dossiers des malades et une fiche d'enquête.

Taille de l'échantillon : recrutement exhaustif des dossiers de tous les patients vus pour traumatisme crânien durant les 3 ans de l'étude soit 21 cas /1304 admissions en neurochirurgie.

Ont été inclus dans l'étude tous les patients de tout âge, de tout sexe, et toute provenance ayant développé au moins deux (2) crises épileptiques après le 7 ème jour suivant leur TC sans ou avec traitement antiépileptique.

Des crises survenues lors des troubles métaboliques, Tous dossiers incomplets (types de crises, mode de survenue, le traitement)

Les tests statistiques descriptives comme les comparaisons (Khi 2), les calculs de fréquence et moyenne ont été faits.

Les informations recueillies ne permettront pas de remonter à l'identité des malades car nous n'avons utilisé que les dossiers des patients avec l'approbation de l'hôpital de Donka. La confidentialité a été gardée sur les dossiers exploités.

RESULTATS :
L'enquête a concerné 21 dossiers de malades hospitalisés pour traumatisme cranio encéphalique ayant développé une Epilepsie Post Traumatique.

Les AVP ont constitué les circonstances de survenue les plus importantes $(81 \%$ dont $52,4 \%$ par Moto, $19 \%$ automobile) représentant $71,4 \%$ des EPT (tableau I). Nous avons reçu $52,4 \%$ des patients avec un Trauma crânien sévère.

L'étude a montré une prédominance masculine $66,7 \%$ avec un sex ratio de 2

Les élèves et étudiants étaient les plus touchés avec $47,6 \%$ (tableau II).

La prévalence de l'EPT était de $1,6 \%$ dans la population hospitalisée et les patients avaient un âge compris entre 2 et 65 ans avec une médiane de 24,59 ans.

A l'admission $90,5 \%$ des patients avaient un trouble de la vigilance, $57,1 \%$ une crise tonico-clonique (tableau III), $61,9 \%$ un point d'impact frontal (tableau IV) et $85,7 \%$ une anomalie focalisée à l'EEG.

La totalité des patients a bénéficié d'un traitement médical et $95,2 \%$ ont eu une amélioration de leur état clinique avant la sortie.

Dans notre étude $52,4 \%$ des patients ont eu un intervalle du séjour de 15 à 30 jours

\section{DISCUSSION}

Les limites principales de ce travail ont été l'inaccessibilité financière de certains patients à l'imagerie par résonnance magnétique, à l'EEG / scanner cérébral et l'insuffisance de suivi des dossiers.

Dans notre étude $81 \%$ des patients ont fait une Epilepsie après un AVP dont 71,4\% par automobile et moto. Ces chiffres pourraient s'expliquer d'une part par le non-respect du code de la route, la non utilisation de ceinture de sécurité par les usagers ainsi que leur imprudence et d'autre part par le manque de panneau de signalisation sur les routes et leur dégradation. Ceci est concordant avec les résultats de Richard I et coll. (9) au service de rééducation fonctionnelle de CHR Nantes /France en 1998.

Le TC sévère après un AVP était de $52,4 \%$. Cette fréquence élevée serait probablement due à la violence du choc pendant l'accident et à la petite taille de notre échantillon. Ce résultat est discordant avec Gueguen B et coll. (10) qui trouve $11,5 \%$ pour le traumatisme crânien grave après un AVP.

La prédominance masculine à $66,7 \%$ s'expliquerait par la nature des activités menées par les hommes les exposant surtout aux AVP et blessures par arme à feu.

Ces résultats concordent avec DIALLO TM et coll. (7) en Guinée en 2004 qui trouvent 25 hommes contre 17 femmes, Richard I et coll. (9) rapportent 75 hommes contre 15 femmes, Wafae El et coll. (5) au Maroc en 2012 démontrent que les EPT dues aux AVP surviennent chez les adolescents et adultes jeunes de 15 à 25 principalement chez les hommes.

Notre étude a trouvé que les élèves létudiants ont été les plus touchés $47,6 \%$ probablement dû à l'exposition de cette couche aux AVP qui sont les étiologies les plus probables des EPT dans la population civile. 
La prévalence de l'EPT était à 1,6\% dans la population hospitalisée pourrait s'expliquer par la courte durée d'hospitalisation des patients ne permettant pas à un nombre plus élevé de patients de développer l'EPT en milieu hospitalier. DIALLO TM et coll. (7) en Guinée en 2004 rapportent 7,1\%, Garga N et coll. (8) en 2006 et Wafae EL (5) au Maroc en 2012 trouvent 20\% d'EPT dans la population générale.

Le trouble de la vigilance lié aux AVP était présent chez $90,5 \%$ des patients car les conducteurs à deux roues ne portent généralement pas de casque, ceux du véhicule pas de ceinture de sécurité et la tête est la plus exposée lors des AVP. Ces résultats sont approuvés par plusieurs auteurs Wafae El et coll. [(5) démontrent que l'incidence de l'EPT augmente de 15 à $30 \%$ selon que le coma est initial, quel que soit sa profondeur et dure une semaine \pm 3 semaines. Gueguen B (11) au centre hospitalier Sainte Anne de Paris en 2009 trouve le coma comme meilleur facteur prédictif d'EPT, qui fait augmenter son incidence de $15 \%$ si le coma dure plus de trois semaines.

Nous avons répertorié $57,1 \%$ cas de crises tonicocloniques nettement supérieur à celui de DIALLO TM et coll. (7) en Guinée en 2004 qui rapportent $35,7 \%$ de crises tonico-cloniques.

Le point d'impact le plus représenté était la région frontale avec $61,9 \%$ qui serait due à l'exposition de cette région lors des AVP d'une part, et son caractère très épileptogène d'autre part. Ce résultat est contraire à celui de Gueguen $B$ (10) qui rapporte une incidence plus élevée de la région pariétale que celle frontale ou temporale.

L'EEG a représenté $85,7 \%$ des bilans en imagerie due à son intérêt dans le diagnostic, la prise en charge, et le suivi de l'EPT. Ce résultat est similaire à celui de Tatai $\mathrm{Al}$ (11) en Algérie en 2012 qui trouve 89\% d'EEG comme examen réalisé sur 100 cas.

Nous avons eu une nette amélioration de l'état clinique avant la sortie chez $95,2 \%$ des patients pouvant s'expliquer par la mise en route du traitement médical bien conduit et/ ou chirurgical d'urgence dès l'admission. La littérature parle de $70 \%$ des épilepsies contrôlées par le traitement médical (12).

\section{CONCLUSION :}

L'EPT est une complication fréquente après un TC sévère et les accidents de la voie publique occupent une grande place dans sa survenue. Le traitement antiépileptique chez un traumatisé crânien en période aiguë peut éviter les complications mais inutile pour prévenir la survenue d'une épilepsie. L'élément préventif sûr de l'Epilepsie Post Traumatique reste la lutte contre les Traumatismes crâniens en insistant sur l'éducation routière, en impliquant les médias dans la stratégie de sensibilisation et en améliorant les conditions de sécurité routière.

Nos remerciements aux responsables de l'hôpital de Donka en Guinée, au personnel enseignant du DERSP du Mali. Mention spéciale au professeur Akory AG IKNANE qui nous a appris la rédaction scientifique / Directeur de l'Institut de Santé Publique du Mali.
Aucun conflit d'intérêt n'est lié au présent travail

\section{REFERENCES :}

Thivard L. Unité d'épileptologie et service de soins de suite et réadaptation neurologique du Pr Baulac, la Salpetrière, Paris ; 2004-12.

2. Maillard L, Michael S, Raffo E, Ducrocq $X$, Vespignani $\mathrm{H}$. Complications traumatiques des crises épileptiques Epilepsie volume 14 Numéro 147-54 Mars 2002.

3. Weiss G H ET COLL. Arch Neurol 1983; 40: 7-10.

4. Annerger J F et coll. Neurology $1980 ; 30: 683-689$.

5. Wafae EL, Nissrine L, Kissani N, Said Ait ben A L'épilepsie post traumatique à Marrakech dans North African and middle east epilepsy journal volum 1 number 2 march- april 2012

6. Dramou b. Profil des cas de TCE et VM dans le service de neurochirurgie de Donka 2001

7. Diallo TM, cisse A, Morel Y, Cisse AF, Souare IS. Premières crises épileptiques tardives étude de 42 cas méd trop 2004.

8. Garga N, Daniel HL L'épilepsie post-traumatique : un problème majeur en besoin urgent d'avancées majeures, L'épilepsie Curr 2006 Janvier, 6 (1): 1-5.

9. Richard I ET coll. Ann Réadaptation Med physique 1998, $41: 409-415$

10. Gueguen B. Epilepsie Post-Traumatique Centre Hospitalier Sainte-Anne Paris S. Guillou révue française 2009

11. Tatai AI Profil de l'épilepsie à Bechar en Algérie étude d'une cohorte de 100 cas dans North African and middle east epilepsy journal volum1 number2 march- april 2012

12. www.who.int/mentalheath/media/en/80/pdf

Liste des tableaux :

Tableau I : Répartition des patients accidentés selon la circonstance de survenue de l'accident à l'hôpital de Donka en 2012

\begin{tabular}{lcc}
\hline Circonstance de survenue & Nombre & $\%$ \\
\hline Accident domestique & 1 & 4,8 \\
Agression physique par arme à feu & 1 & 4,8 \\
AVP auto & 4 & 19,0 \\
AVP moto & 11 & 52,4 \\
AVP vélo & 2 & 9,5 \\
Chute & 2 & 9,5 \\
Total & 21 & 100 \\
\hline
\end{tabular}

Tableau II : Répartition des patients selon leur profession

\begin{tabular}{lcc}
\hline Profession & Nombre & $\%$ \\
\hline Elèves /Etudiants & 10 & 47,6 \\
Fonctionnaire & 1 & 4,8 \\
Profession libérale & 8 & 38,1 \\
Autre & 2 & 9,5 \\
Total & 21 & 100 \\
\hline
\end{tabular}

Autre : ménagère et sans profession 
Tableau III : Répartition des patients accidentés selon le type de crises à l'hôpital de Donka en 2012

\begin{tabular}{lll}
\hline Type de crise & Nombre & $\%$ \\
\hline Clonique & 2 & 9,5 \\
Tonico-clonique & 12 & 57,1 \\
Tonique & 2 & 9,5 \\
Myoclonique & 1 & 4,8 \\
Association & 4 & 19,0 \\
\hline
\end{tabular}

Tableau IV : Répartition des patients accidentés selon le point d'impact du trauma crânien à l'hôpital de Donka en 2012

\begin{tabular}{lll}
\hline Point d'impact du TC & Nombre & $\%$ \\
\hline Frontal & 13 & 61,9 \\
Temporal & 5 & 23,3 \\
Pariétal & 5 & 23,3 \\
Occipital & 3 & 13,3 \\
\hline
\end{tabular}

